\title{
Statistical Properties of Random Packing and Macroscopic Thermal Conductivity of Sintered Balloons*
}

\author{
(Re-Examination)
}

\author{
Isao TAGUCHI $^{* *}$ and Michio KURASHIGE ${ }^{* * *}$ \\ ${ }^{* *}$ Aomori Prefectural Agriculture and Forestry Research Center, \\ Field Crops and Horticulture Experiment Station, \\ Inuotose-Yanagisawa 91, Rokunohe, Aomori, 033-0071, Japan. \\ E-mail: isao_taguchi@pref.aomori.Ig.jp \\ *** Department of Mechanical Engineering, Iwate University, \\ Ueda 4-3-5, Morioka-shi, Iwate 020-8551, Japan.
}

\begin{abstract}
This paper simulates the macroscopic thermal conductivities of randomly-packed, sintered balloons for various degrees of sintering and for a wide range of the balloon inner/outer diameter ratio. Although this problem was already studied by Ono et al., we found some errors in their paper, including a critical one. Thus, we re-examine the same theme and correct all errors. Furthermore, we extend its contents and discuss the results in more detail. For practical purposes, we present all the macroscopic conductivities in terms of a simple expression of two balloon's geometrical parameters. "Specific" macroscopic thermal conductivities (i.e., those per weight) are precisely estimated and found to have a peak when the balloons have the diameter ratio larger than approximately 0.8 .
\end{abstract}

Key words: Simulation, Balloon, Thermal Conductivity, Random Packing, Anisotropy

\section{Introduction}

Porous materials such as a foam and sintered powder have complicated and heterogeneous microstructures, which represent superior properties and functions in many cases. Thus, they have a wide range of applications. From a topological point of view of the microstructures, these materials can be divided into two groups: closed-cell foam and open-cell foam. ${ }^{(1)}$

Closed-cell foams have high modulus and strength characteristics or high impact-energy absorbing characteristics. Open-cell foams are neither as stiff nor as strong as the closed ones. However, they possess various multifunctional characteristics, since they have an ability to flow fluids readily through open pores. They are used as filters and catalyst supports. The mechanical properties of the closed-cell foams are lower than these by the theoretical prediction; wrinkles in the cell walls reduce their measured properties to values similar to those for the open-cell foams. ${ }^{(2),(3)}$

In recent years, sintered compacts of balloons made of ceramics, metal, etc, have started to become commercially available. Bonded compacts of hollow spheres may be good alternative and new category materials, because they have both pore characteristics of openand closed-cells. ${ }^{(5)-(8)}$

Such functions of these porous materials are related to internal heterogeneous 
microstructures. Understanding the relationship between the microstructures and macroscopic properties can allow the materials design. To understand it, it is necessary to establish an evaluation method for macroscopic properties of sintered balloons.

Ono et al. ${ }^{(9)}$ applied a three step simulation method to evaluation of thermal conductivity of sintered balloon aggregate. This simulation method, which is composed of "geometrical model construction in a computer", "finite element analysis of an extracted particle pair", and "structural analysis of a random network", was proposed by Kurashige et al. ${ }^{(10)}$ and Kato et al. ${ }^{(11)}$ to predict effective elastic moduli of randomly packed identical sphere aggregates. They reconfirmed that the packing constructed under gravity is of transverse isotropy, and quantitatively analyzed randomness of the packing from a statistical parameter representing the degree of crystallization. Furthermore, they estimated effective thermal conductivities of the "sintered" balloons.

However, we found some errors in their paper ${ }^{(9)}$, including a critical one. Thus, we re-examine the same theme and correct all errors.

Furthermore, we extend its contents to a wide range of the ratio of their inner diameter to outer one in consideration of commercially available balloons. ${ }^{(6)}$ For practical purposes, we present all the macroscopic thermal conductivities in terms of a simple expression of two balloon's geometrical parameters. We precisely estimate "specific" macroscopic thermal conductivities (i.e., those per weight), and discuss the features of the sintered balloon aggregates in more detail.

\section{Simulation Procedures}

In order to investigate microstructures of a balloon aggregate, we carry out the random packing simulation of sequential accumulation of identical spheres into a virtual box, and examine statistical characteristics of the packings such as porosity and coordination number.

Next, we extract a pair of connected balloons from the packing and regard it as a resistor. The thermal resistance of the resistors is calculated by the finite element analysis.

Finally, we replace the aggregate by a "random network" of resistors with the resistance estimated in the above. We assign temperatures at all nodes near the ends of the network and determine temperatures in steady state. The macroscopic thermal conductivity can be obtained by the temperature difference between the opposite cube surfaces and the total flow rate.

\section{Geometrical Model of Random Packing}

\subsection{Equation of motion}

To generate a geometrical model of three-dimensional sphere aggregate in a computer, we perform a random packing simulation. We sequentially throw identical spherical particles with diameter $D$ from random positions $(x, y)$ into a virtual box; we take the $z$ direction in the direction of gravity. For the sake of simplicity, we assume that a falling particle is subjected to only gravity and forces from resting particles when collided with them. Ignoring an acceleration term, we can express the motion equation of the particle as follows:

$$
c \frac{d \boldsymbol{x}}{d t}=\sum k \Delta \boldsymbol{d}-m g \boldsymbol{k}
$$

where $\boldsymbol{x}$ is the position vector of a falling sphere's center, $\boldsymbol{k}$ is the unit vector along the $z$ axis and $\Delta \boldsymbol{d}$ is the overlapping depth vector of the particle in collision with another still one. Notations $c, k, m$ and $g$ are the viscosity constant, the spring constant between two particles in collision, the mass of each sphere and the gravitational acceleration, respectively. 
Summation notation $\sum$ is over the number of all particles in contact with the one under consideration.

Using balloon's outer diameter $D$ as a reference length, we introduce non-dimensional variables and constant parameters: $\boldsymbol{X}=\boldsymbol{x} / D, \Delta \boldsymbol{D}=\Delta \boldsymbol{d} / D, T=m g t /(c D), K=D k /(m g)$. Thus, Eq. (1) can be rewritten in terms of these as follows:

$$
\frac{d \boldsymbol{X}}{d T}+\sum K \Delta \boldsymbol{D}-\boldsymbol{k}=0
$$

We use the Runge-Kutta-Gill method to solve the three coupled differential equations. We calculate coordinates of the balloon's center at discrete time with time increment $\Delta T$. If the distance over which the balloon moves during $\Delta T$ is smaller than some given small value $\varepsilon$, we judge that it has stopped and then throw a new balloon into the box. The settled spheres never move even if they are hit by a new sphere. In running our program, we use the following numerical value: time increment $\Delta T=1.0 \times 10^{-3}$, spring constant $K=1.0 \times 10^{3}$ and small parameter $\varepsilon=1.0 \times 10^{-7}$, which were employed in Ono et al. ${ }^{(9)}$

\subsection{Simulation Method}

Consider a virtual box of $0 \leq x \leq B, 0 \leq y \leq B, 0 \leq z \leq H(B<H)$. We do not stop introducing particles until the region $0 \leq x \leq B, 0 \leq y \leq B, 0 \leq z \leq B$ is completely filled with particles. We adopt box sizes $B / D=20$ and ten runs are made with ten different sets of pseudorandom numbers.

We use the periodic boundary conditions and displace the floor up and down in order to avoid packing regularity on its walls and floor, respectively. These random numbers are generated by the algorithm of Wichmann and Hill. ${ }^{(12)}$

\section{Statistical Properties of Random Packing}

\subsection{Porosity}

An averaged porosity of the random packing constructed by the above method is 0.417 and the standard deviation is 0.00065 , which is small enough.

Using these results, let us discuss the validity of the packing method and the geometrical model. In general, random packings are separated into three categories. (13),(14) "Close random packing" is produced after the container is well shaken, i.e., when there is considerable momentum and little chance of bridging. The porosity is $\phi \approx 0.36$. "Loose random packing" $(0.41 \leq \phi \leq 0.44)$ is obtained by dumping and in some cases by tapping the container, which gives the spheres more momentum but provides little opportunity for bridging. "Very loose random packing" is obtained when momentum is minimized and opportunities for bridging are maximized. For example, when the fluid velocity in a fluidized bed is slowly reduced, spheres settle to a mean porosity of approximately $0.44 \sim 0.47$.

The porosity $\phi=0.417$ obtained here is rather small in the range of loose random packing, because the present method allows no bridging. Therefore, this simulation method reflects reality quantitatively well in spite of its simplicity.

\subsection{Coordination Number}

A coordination number is defined as the number of contact points of a particle with neighboring ones in a regular or irregular packing of particles. For a particle aggregate, electric current or heat is conducted through those contact points. Therefore, it is important to evaluate the coordination number to know thermal conductivities of the particle aggregate.

With a few regular packings, it is six for a simple cubic, 8 for a body-centered cubic and 12 for a face-centered cubic packings; in contrast, the random packing has a possibility that a 
sphere is in contact with 4 to 12 other spheres and its average coordination number has a peak at approximately six for the random packing.

The mean coordination number over the ten samples is 5.983 with standard deviation of 0.0019 , which may be small enough.

Ono et al. ${ }^{(9)}$ showed that the mean value was 6.017 . However, it is found that there are some mistakes in counting the contact points near the upper and lower surfaces. Thus, we correct their error in the mean coordination number; the correct one created by the present algorithm is 6.019 .

As in the previous paper, we define a "branch" as a line segment connecting the centers of two spheres in contact or almost in contact. We judge that the two spheres are in contact if the branch length is smaller than sphere diameter $D$ as reference length. However, this judging is very delicate; for example, the coordination number depends on how to choice $\Delta T$. Therefore, it may be required to check how the coordination number changes according to the criterion, i.e., the reference length $R L$, for the judge. Changing the reference length from $0.99 \mathrm{D}$ to $1.01 \mathrm{D}$, we calculate an average coordination number changing the judgment length from $0.99 D$ to $1.01 D$. The result averaged over ten samples is shown in Fig. 1. It can be seen that the contacts occur approximately at $R L=0.997 D$ and they rapidly increase from $R L=0.990 D$ to $1.0 D$; in other words, the shortest branch is approximately $0.997 D$, and there are most contact points between $R L=1.0 D$ from $0.999 D$. Thus, the present judge is good in contact point of view.

Furthermore, we consider the relationship between the coordination number and porosity. The mean coordination number of the present random packing is 6.019 , which is close to the coordination number of the simple cubic packing. In contrast, the porosity of the present random packing differs from that of simple cubic packing. The reason can be as follows. The simple cubic packing is in unstable equilibrium, i.e., an upper sphere is right above a lower sphere. The state gets energetically more settled by the slight side move of the upper layer. Because the upper layers sink, the porosity becomes small, but the coordination number does not change very much in this process. Since the present random packing is a stable structure, its porosity is considerably small, but its coordination number is close to that of the simple cubic.

The porosity 0.417 obtained hear is rather small in the porosity range of loose random packing; this may be come from the sequential deposition method used, which allows no bridging.

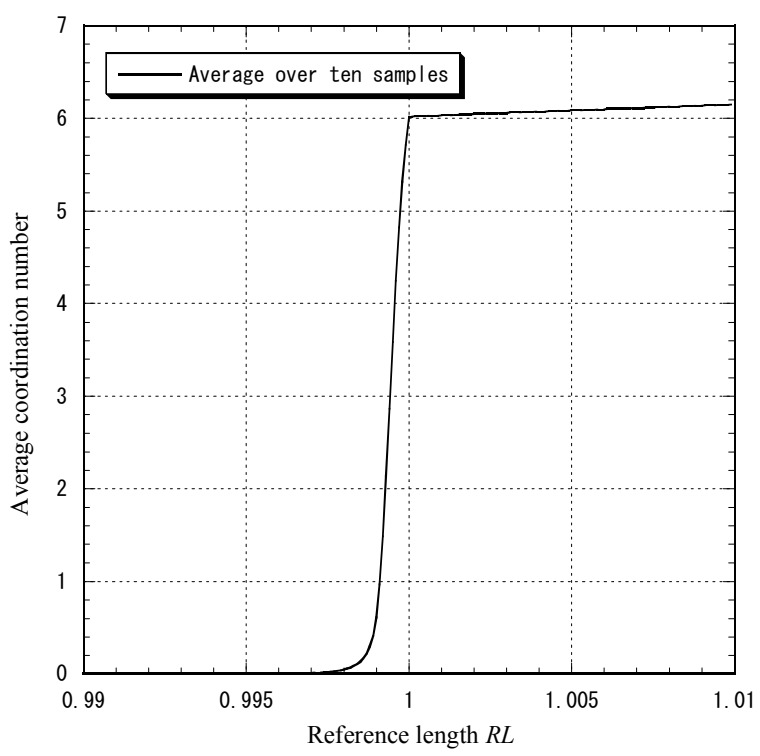

Fig. 1 Average coordination number versus reference length 


\subsection{Effect of Gravity}

Kato et al. ${ }^{(11)}$ and Ono et al. ${ }^{(9)}$ examined how the branch orientations distribute. It was found that line segments connecting the centers of the balloons in contact lie more frequently around the direction of 51 degrees from the vertical line, while they are uniformly distributed about the horizontal line. Since this is a crucial result related to the microstructure and macroscopic property, the present result is shown in detail in what follows.

We divide the domain of zenithal angle $\theta,(0, \pi / 2]$, into nine sub-domains. Here, the zenithal angle is defined by the angle measured from the vertical axis. It could be seen that the frequency distribution is not uniform at all; the maximum frequency is approximately $18 \%$ in the $50^{\circ}-60^{\circ}$ direction, while the minimals are approximately $5 \%$ in the $0^{\circ}-10^{\circ}$ and $80^{\circ}-90^{\circ}$ directions. Assuming that the frequency distribution of branches is an even function both about $\theta=0^{\circ}$ and $\theta=90^{\circ}$, we can express the distribution by the Fourier series of $\cos 2 m \theta$. If we take only the first four terms, the distribution is as follows:

$$
\begin{aligned}
& F(\theta)=\sum_{m=0}^{3} a_{m} \cos 2 m \theta \\
& a_{0}=11.11, a_{1}=-1.49, \quad a_{2}=-6.11, a_{3}=1.40
\end{aligned}
$$

These coefficients have been determined by the least square method. It can be seen that the frequency distribution has its peak at $\theta=50.9^{\circ}$. If we make the major diagonal line of a simple cubic lattice to the vertical direction, the zenithal angle of the lattice orientation is as follows:

$$
\theta=\operatorname{Cos}^{-1}\left(\frac{1}{\sqrt{3}}\right)=54.7^{\circ} .
$$

Therefore, we can say that the packing structure obtained by the present method is similar to that of the simple cubic lattice, as the coordination number is.

This agreement shows that the characteristics of branch distribution in the horizontal plane are isotropic but different from those in the vertical direction. The distribution of branch orientations on all directions would be the same if the packing structure were "perfectly" random. Therefore, we could say that the packing structure has regularity from the branch orientations point of view.

\section{Evaluation of Macroscopic Thermal Conductivity of Sintered Balloons}

We regard the geometrical model created by the above random packing simulation as a random network of resistors, whose thermal resistance is evaluated by the finite element method. Applying different temperatures to the end of the network, we calculate the macroscopic thermal conductivity of the aggregate from the total heat flux and node temperatures at all ends of the resistors.

\subsection{Geometry of a Sintered Balloon Pair}

We will estimate the thermal resistance ratio $K / K_{0}$ of a sintered half-balloon pair picked out from the aggregate by the finite element analysis; $K_{0}$ is the inverse of the axial thermal resistance of a cylindrical solid bar with the same diameter and length as the outer diameter of a balloon, while $K$ the counterpart of the sintered half-balloon pair.

The model of sintered balloon pair is created to simulate the conductance of balloon pair as shown in Fig. 2, where $D$ and $d$ are the outer and inner diameters of the balloon, respectively. In sintering of the balloon aggregate, necks are created around contact points of 
the balloons by mass diffusion; the necks grow up with sintering time. However, we add, around a contact point of the two half-balloons, some appropriate amount of mass of the same substance as that of the balloons in this paper. We will adopt the angle $\Theta$ as a parameter of the sintering degree. The geometry made by adding the mass is not exactly the same as that of real sintered balloons but, at least, the shape of void space in the mass-added aggregate is used as a model of adhesion, and is much more similar to the real one than the void shape in the self-consistent composite models. ${ }^{(15),(16)}$

As a function of the sintering degree angle $\Theta$, the added volume per contact point can be expressed as follows:

$$
V_{\text {add }}=\frac{\pi}{4}\left(\frac{1-\cos \Theta}{\cos \Theta}\right)^{2}\left\{1-\left(\frac{\pi}{2}-\Theta\right) \tan \Theta\right\} \times D^{3},
$$

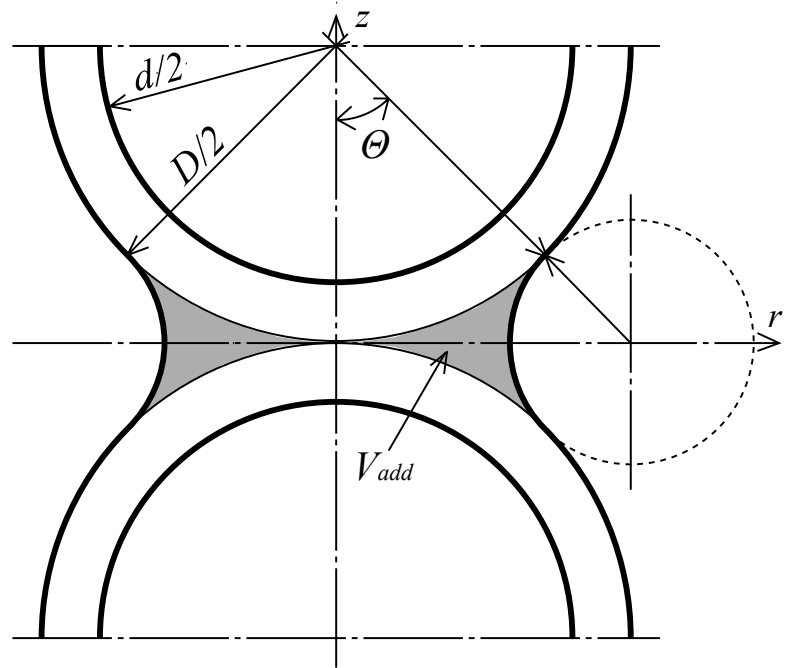

Fig. 2 Geometry of sintered (mass-added) and hollow hemispheres

\subsection{Simulation method}

Let us consider three-dimensional heat conduction in the sintered pair of balloons, whose geometry is shown together with the cylindrical coordinate system $(r, z)$ in Fig. 2 . In the analysis, neither heat conduction nor convection in and no radiation into the vacant spaces of the sintered balloons are assumed, but only the heat conduction through the solid matrix of the aggregate is taken into account. This is true in the present case the thermal conductivity of balloon materials is much higher than that of gases. The conductivity of the added volume material is assumed identical to that of balloon substance.

The boundary conditions at both ends $z= \pm D / 2$ of the pair are given by $T= \pm \Delta T_{0} / 2$, respectively, and the outer and inner surfaces of the pair are insulated, that is, $\partial T / \partial n=0$ on these surfaces; $T$ and $n$ are the temperature and the outward unit normal to them. For the finite element analysis, an arbitrary cross section containing the $z$-axis is divided into finite triangles, leading to triangular ring elements because of the axisymmetric geometry. Numbers of nodes and elements are 2640 and 4949, respectively, for the thickest-walled balloons, while the thinnest-walled model contains 9980 nodes and 18545 elements.

As the results of the finite element analysis, it could be seen that the inverse resistance ratios monotonically increase with increasing the sintering degree angle, seeming to converge to zero with the vanishing angle. The ratios are very sensitive to changes in the sintering degree when the angle is small; the slight increase of the contact area from a point contact causes the sharp rise in the heat flux. 


\subsection{Random Network of Thermal Resistors}

To evaluate the macroscopic thermal conductivity of the sintered balloon aggregate, we will replace the aggregate by a "random network" of resistors with the resistance estimated in the above.

The first step is to construct each element stiffness matrix in the network. Next, to find the global equation system for the whole solution region, we must assemble all the element equations.

We assign temperatures at the nodes (or resistors' ends, which are originally situated at the balloons' centers) existing within the outermost layers of thickness $D / 2$ in a cubic box webbed by resistors; the values of these assigned temperatures are determined so as to be proportional to the corresponding values of coordinate in the macroscopic heat flow direction, with the temperatures being zero and a given $T_{0}$ at the two opposite surfaces of the cube. The other four surfaces are assumed to be insulated. Thus, the law of Kirchhoff determines temperatures at all nodes.

From the temperatures at all nodes determined as in the above, the total heat flow rate can be estimated and then the macroscopic thermal conductivity is obtained by dividing the total flow rate by the temperature difference between the opposite cube surfaces.

\section{Results and Discussion}

\subsection{Macroscopic thermal conductivity (correction)}

First of all, we will point out an error in Figs. 8 and 9 in Ono et al. ${ }^{(9)}$, which was made in calculation of porosity. We show the correct macroscopic thermal conductivity in Figs. 3 and 4 , and discuss the new results as below.

\subsection{Macroscopic thermal conductivity of sintered balloons}

Figure 3 shows the macroscopic thermal conductivities in the $X$ direction of the sintered balloon aggregates for several ratios of the inner/outer diameters $(d / D=0.0,0.5$, $0.6,0.7,0.8$ and 0.9 ) for five samples. In this figure, the ordinate presents their macroscopic thermal conductivity $\kappa$ normalized by that of the balloon substance $\kappa 0$, while the abscissa is for the porosity of the sintered aggregates; the porosity contains hollow volume of the balloons in Fig. 2 as well. Each marker on an individual curve in the figure corresponds to the sintering degree angle ranging from $3^{\circ}$ to $45^{\circ}$ at the interval of $3^{\circ}$ and the progress toward the right on each curve makes the sintering degree low and the porosity increase.

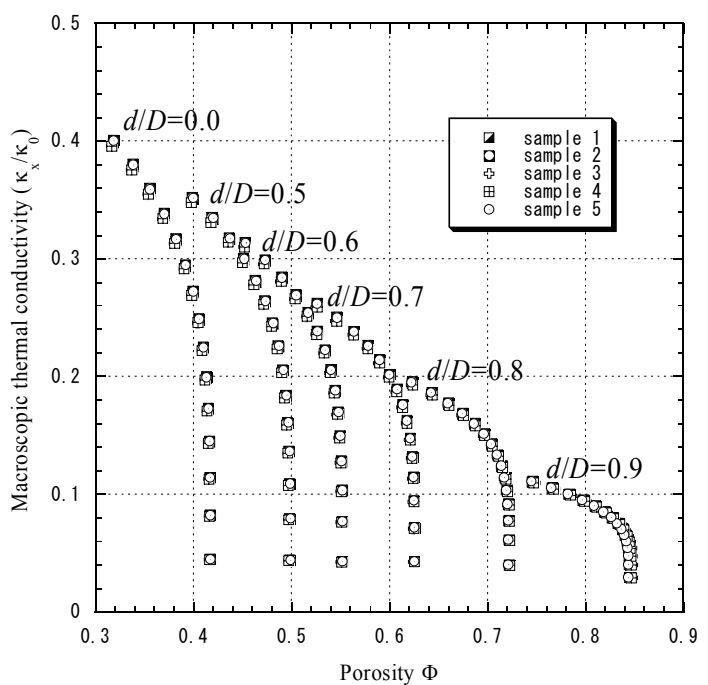

Fig. 3 Macroscopic thermal conductivity along $X$ axis for the first five samples 
We examined dependence of the macroscopic thermal conductivity on the inner-outer diameter ratio. Increases in the diameter ratio lower the conductivity curves as a whole, and shift them toward higher porosity, because the balloon wall becomes thinner. The conductivities are little affected by the ratio if it is for the thick balloon wall $d / D<0.5$. In contrast, the thermal conductivities lower rapidly with the diameter ratio if it is larger than 0.5 .

Next, let us examine dependence of the conductivity on the degree of sintering. For each diameter ratio, the smaller the sintering degrees, the lower the conductivities due to the larger resistance at the necks. The macroscopic thermal conductivity of the hollow sphere with $d / D=0.9$ is approximately a quarter of that of the solid sphere. In contrast, the macroscopic thermal conductivity of the thicker balloon aggregates has little difference.

We will show only the graphs for sample 1 in the following, because of almost vanishing difference among the samples shown in Fig. 3.

Figure 4 shows that the macroscopic thermal conductivities in the $X(4 \mathrm{a}), Y(4 \mathrm{~b})$ and $Z$ (4c) directions, respectively, for the diameter ratios $d / D$ ranging from 0.0 to 0.9 with their small increment 0.1 . The macroscopic thermal conductivity in the $X$ direction is almost exactly equal to that in the $Y$ direction. On the other hand, the macroscopic thermal conductivity in the $Z$ direction (vertical) is approximately $20 \%$ larger than that in $X$ direction (horizontal) over a whole range of diameter ratio. This reveals that the simulated packing structure is of isotropy in the $X-Y$ plane and of anisotropy normal to this plane. The anisotropy of the thermal conductivities may be caused by the deflection in the zenithal distributions of the branches.
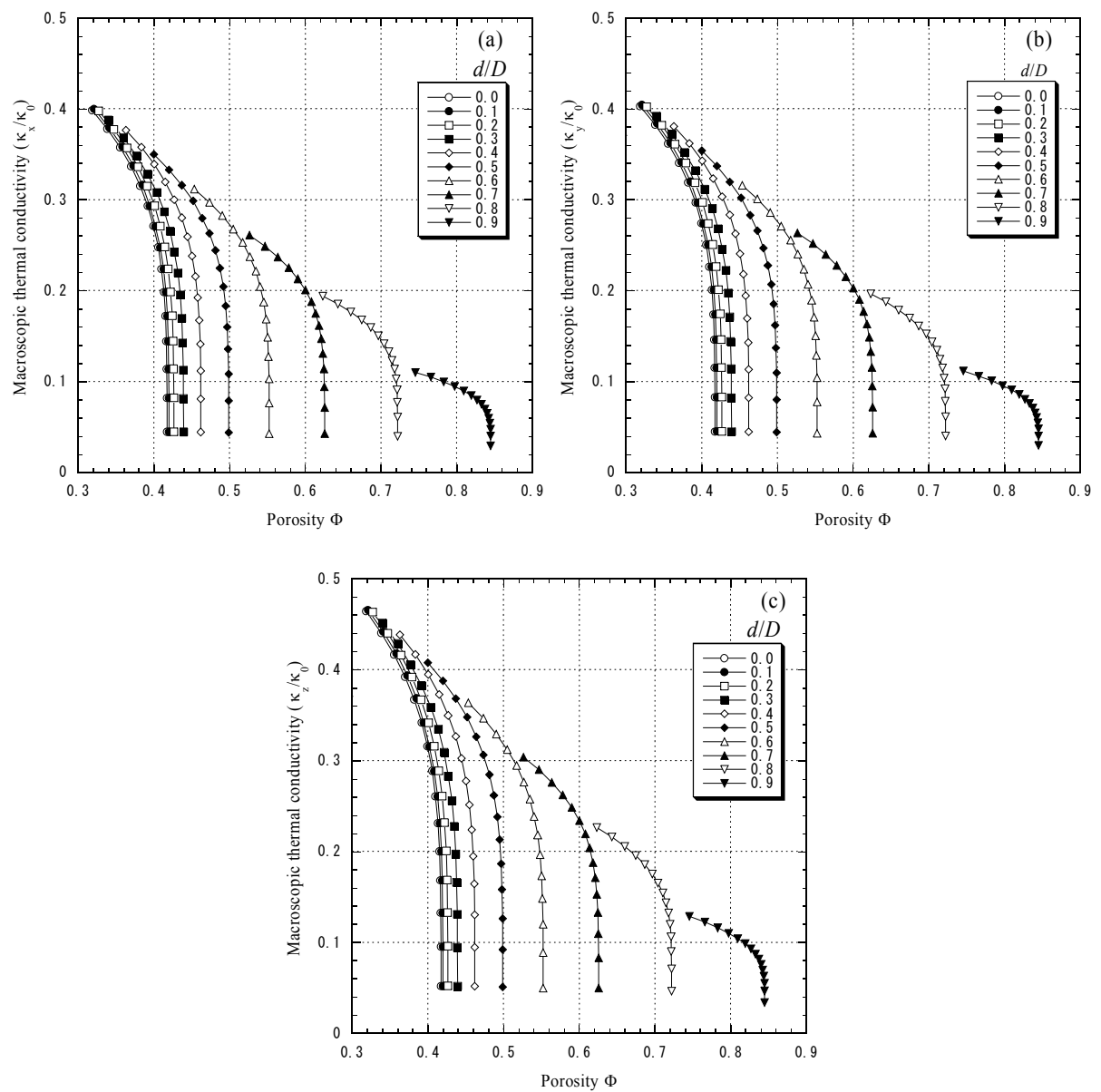

Fig. 4 Macroscopic thermal conductivity along the $X, Y$ and $Z$ axes, (left, right and bottom, respectively) 
From the simulation results, we have derived expressions to predict the thermal conductivities in terms of the two geometrical parameters $d / D$ and $\Theta$.

$$
\begin{aligned}
\frac{\kappa_{x}}{\kappa_{0}} & =\left(1.19 \times 10^{-2}+8.27 \times 10^{-3}\left(\frac{d}{D}\right)-1.74 \times 10^{-2}\left(\frac{d}{D}\right)^{2}\right) \Theta \\
& +\left(-7.66 \times 10^{-5}-9.7 \times 10^{-5}\left(\frac{d}{D}\right)+1.24 \times 10^{-4}\left(\frac{d}{D}\right)^{2}\right) \Theta^{2} \\
\frac{\kappa_{z}}{\kappa_{0}} & =\left(1.39 \times 10^{-2}+9.64 \times 10^{-3}\left(\frac{d}{D}\right)-2.03 \times 10^{-2}\left(\frac{d}{D}\right)^{2}\right) \Theta \\
& +\left(-8.93 \times 10^{-5}-1.13 \times 10^{-4}\left(\frac{d}{D}\right)+1.44 \times 10^{-4}\left(\frac{d}{D}\right)^{2}\right) \Theta^{2},
\end{aligned}
$$

Equations (6) and (7) describe the results for $0.0 \leq d / D \leq 0.9$ and $3^{\circ} \leq \Theta \leq 45^{\circ}$ to within $8 \%$ in all cases.

Furthermore, we have evaluated the macroscopic thermal conductivities for a case of thinner balloon in detail. We will show only the expressions because the results have similar tendency.

$$
\begin{aligned}
\frac{\kappa_{x}}{\kappa_{0}}= & \left(2.36 \times 10^{-1}+2.88 \times 10^{-2} \Theta-1.92 \times 10^{-4} \Theta^{2}\right)\left(\frac{d}{D}\right) \\
& +\left(-2.3 \times 10^{-1}-2.91 \times 10^{-2} \Theta+2.01 \times 10^{-4} \Theta^{2}\right)\left(\frac{d}{D}\right)^{2}, \\
\frac{\kappa_{z}}{\kappa_{0}} & =\left(2.75 \times 10^{-1}+3.35 \times 10^{-2} \Theta-2.24 \times 10^{-4} \Theta^{2}\right)\left(\frac{d}{D}\right) \\
& +\left(-2.68 \times 10^{-1}-3.39 \times 10^{-2} \Theta+2.34 \times 10^{-4} \Theta^{2}\right)\left(\frac{d}{D}\right)^{2}
\end{aligned}
$$

Equations (8) and (9) describe the results for $0.9 \leq d / D \leq 0.95$ and $3^{\circ} \leq \Theta \leq 45^{\circ}$ to within $2 \%$ in all cases. It should be noticed that we have interchanged $d / D$ and $\Theta$.

Feng et al. ${ }^{(18)}$ measured an electrical conductivity of foams with different cell diameter. It was shown that when the samples possess a roughly equal porosity, the electrical conductivities are almost identical among samples. Therefore, they concluded that the cell diameter had a minor influence on the electrical conductivity of foams. However, in Fig. 3, for example, at the porosity of about 0.62 , the thermal conductivities of $d / D=0.7$ and 0.8 are almost the same. In contrast, for the curve of $d / D=0.7$, decreasing sintering degree angle, in other words approaching the point contact, the macroscopic thermal conductivity suddenly falls but the porosity unchanged. Therefore, it is inappropriate to evaluate the macroscopic thermal conductivity only in terms of porosity for the sintered balloons, that is, the macroscopic thermal conductivity depends on the inner-outer diameter ratio (material) and sintering degree angle (sintering method).

\subsection{Specific Macroscopic thermal conductivity}

As mentioned in Introduction, one of the advantageous properties of the sintered balloon aggregates has high specific properties. Thus, we will estimate a specific macroscopic thermal conductivity, which is defined as the macroscopic thermal conductivity divided by effective density of the sintered balloon aggregate $(1-\Phi)$. 

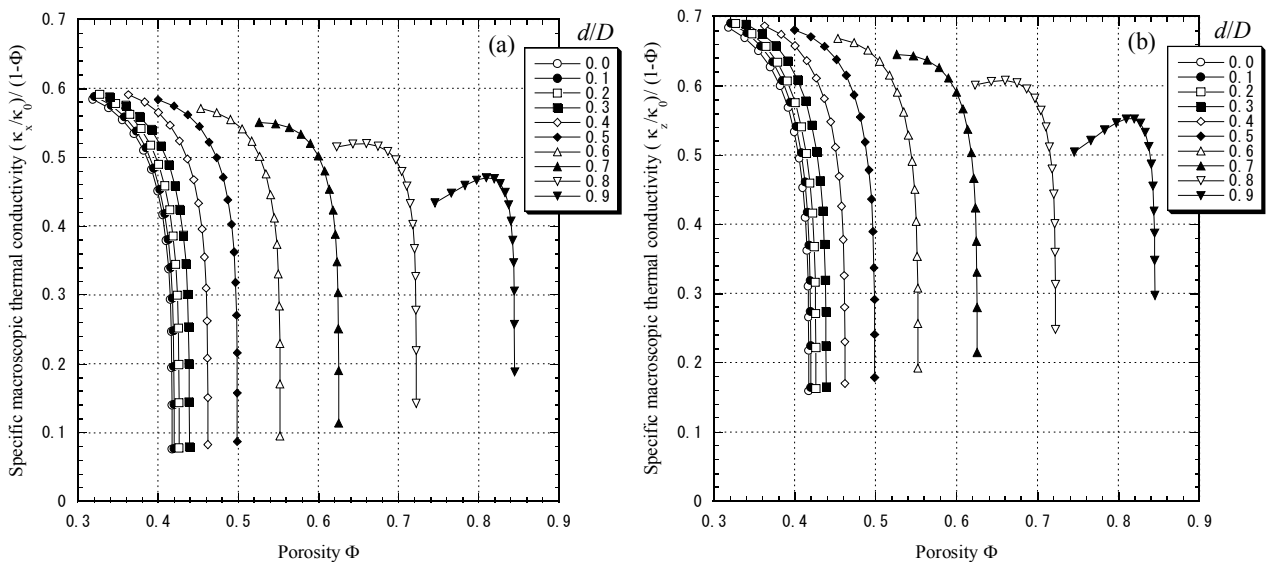

Fig. 5 Specific macroscopic thermal conductivity along the $X$ and $Z$ axes, (left and right, respectively)

Figures $5 \mathrm{a}$ and $5 \mathrm{~b}$ show the specific macroscopic thermal conductivities in the $X$ and $Z$ direction, respectively, for $d / D=0.0$ to 0.9 with the interval of 0.1 . We omit the graph of the results in the $Y$ direction because the results have almost the same results of the $X$ direction. Comparing Fig. 5 with Fig. 4, the effective density decreases as the diameter ratio increases and the degree of sintering declines; that is, the density is lower for the thinner balloon wall and for the less sintered balloon aggregate. Therefore, relatively to the curves in Fig. 4, all the curves as a whole move upwards more for the larger diameter ratio, while further right part of each curve rises for a specified ratio because of the less sintered aggregate or the smaller effective density.
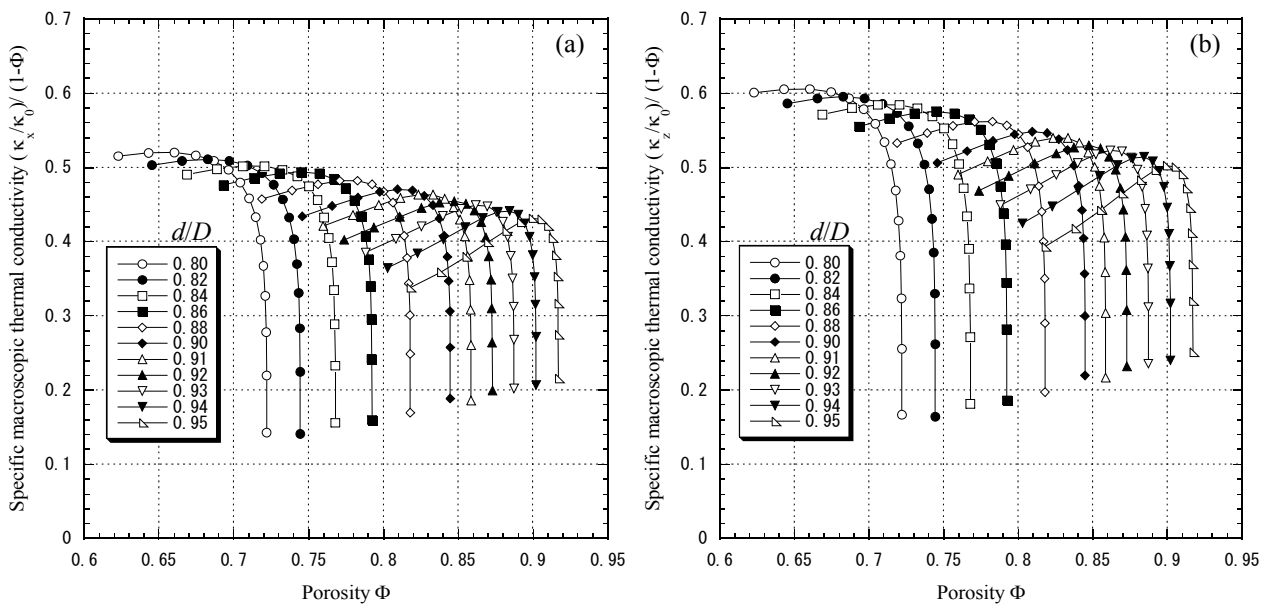

Fig. 6 Specific macroscopic thermal conductivity along the $X$ and $Z$ axes for the range from $d / D=0.8$ to 0.95 , (left and right, respectively)

It can be seen that the specific macroscopic thermal conductivities have a peak for the case of $d / D \geq 0.8$. In order to argue this phenomenon, we have calculated the specific conductivities for the case of $d / D \geq 0.8$ in detail and shown them in Fig. 6. For $d / D=0.8$, the specific conductivity have a peak at approximately $\Theta=40^{\circ}$, while at approximately $\Theta=25^{\circ}$ for $d / D=0.95$. The larger the inner-outer diameter ratio is, the smaller the peak angles are. The peak angles are sharper for the thinner balloons. In order to physically explain the phenomenon, we introduce effective sectional area $A_{\text {eff }}$, which is defined by $A_{\text {eff }}=A_{\text {outer }}-$ Ainner ; here Aouter and Ainner imply sectional area of outer and inner of the balloon, respectively. For the case of solid particle aggregate, because the sectional area of the neck is much smaller than that of particle, the overall thermal conductivity of the sintered 
aggregate is dominated by the sectional area of the neck; the neck growing leads to increase in the macroscopic conductivity. In contrast, for the case of thin balloon aggregate, the cross sectional area of the neck is larger than the effective cross sectional area of the balloon in some cases. In this case, the increase in the cross-sectional area of the neck contributes less to increasing the macroscopic thermal conductivity in spite of the increase of the mass. For the case of thinner balloons, the specific conductivities have a peak at the small sintering angle, whose peak is sharp because the effective cross sectional area is small. In the material design, therefore, we must select the best sintering condition from this result.

\section{Conclusions}

Using a three-step simulation method which composed of "geometrical model construction," "finite element analysis of balloon pair" and "simulation of macroscopic property," we have re-evaluated the macroscopic thermal conductivities of the sintered, randomly packed balloons, which is the same theme as in Ono et al. ${ }^{(9)}$ and have corrected all their errors.

The important findings obtained through this study can be summarized as follows:

(1) For practical purposes, we present all the macroscopic thermal conductivities by a simple expression of two balloon's geometrical parameters. It has been shown that the macroscopic thermal conductivity cannot be evaluated only in terms of the porosity for the sintered balloons.

(2) Specific macroscopic thermal conductivities have been precisely estimated. It has been found that the specific conductivities have a peak when the balloons have the diameter ratio larger than approximately 0.8 . For the lager inner-outer diameter ratio, and the smaller sintering degree angle, the peaks appear and are sharp. Using the effective sectional area of balloons and sectional area of a neck, we could show why the peaks appear.

\section{References}

(1) Gibson, L.J. and Ashby, M.F., Cellular Solids: Structure and Properties, Second Edition, (1997), Cambridge University Press, Cambridge.

(2) Simone, A.E. and Gibson, L.J., Effects of Solid Distribution on the Stiffness and Strength of Metallic Foams, Acta Materialia, Vol. 46 (1998), pp. 2139-2150.

(3) Andrews, E., Sanders, W.S. and Gibson, L.J., Compressive and Tensile Behavior of Aluminum Foams, Materials Science and Engineering A, Vol. 270 (1999), pp. 113-124.

(4) Norris, B. and Gojny, F.J., Hollow Metal Sphere Filled Stabilized Skin Structures and Method of Making, United States Patent, No. 4925740, 15 May (1990).

(5) Andersen, O., Wang, U., Schnerder, L., Stephani, G. and Kieback, B., Novel Metallic Hollow Sphere Structure, Advanced Engineering Materials, Vol. 2-4 (2000) pp. 192-195.

(6) Sanders, W.S. and Gibson, L.J., Mechanics of Hollow Sphere Foams, Materials Science and Engineering A, Vol. 347 (2003), pp. 70-85.

(7) Gasser, S., Paun, F., Cayzeele, A. and Bréchet, Y., Uniaxial Tensile Elastic Properties of a Regular Stacking of Brazed Hollow Spheres, Scripta Materialia, Vol. 48 (2003), pp. 1617-1623.

(8) Queheillalt, D.T., Sypeck D.J. and Wadley, H.N.G., Ultrasonic characterization of cellular metal structures, Materials Science and Engineering A, Vol. 323 (2002), pp. 138-147.

(9) Ono, K., Kurashige, M. and Imai, K., Statistical Properties of Equal-Sized Sphere Random Packing and Conductivity of Sintered Balloons, Transactions of the Japan 
Society of Mechanical Engineers, Series A, Vol. 70, No.689 (2004), pp. 126-133.

(10) Kurashige, M., Hayashi, T. and Imai, K., Simulated Effective Elastic Moduli and Wave Velocities in Water-Saturated Sintered Glass-Beads, Acta Mechanica, Vol. 132 (1999), pp. 177-194.

(11) Kato, H., Matsunaga, C., Kurashige, M. and Imai, K., Anisotropy in Packing Structure and Elasticity of Sintering Spherical Particles, JSME International Journal, Series A, Vol. 45-4 (2002), pp. 585-595.

(12) Wichmann, B.A. and Hill, I.D., An Efficient and Portable Pseudo-Random Number Generator, Royal Statistical Society, Algorithm as 183 (1982), pp. 188-190.

(13) Haughey, D.P. and Beveridge, G.S.G., Structural Properties of Packed Beds, The Canadian Journal of Chemical engineering, Vol. 47 (1969) pp. 130-140.

(14) Tory, E.M., Church, B.H., Tam, M.K. and Ratner, M., Simulated Random Packing of Equal Sphere, The Canadian Journal of Chemical engineering, Vol. 51 (1973), pp. 484-493.

(15) Nolan, G.T. and Kavanagh, P.E., Computer Simulation of Random Packing of Hard Spheres, Powder Technology, Vol. 72 (1992), pp. 149-155.

(16) Hill, R., Self-Consistent Mechanics of Composite Materials, Journal Mech. Phys. Solids, Vo. 13 (1965), pp. 213-222.

(17) Taya, M. and Arsenault, R.J., Metal Matrix Composite, (1989), pp. 197-198, Pergamon Press.

(18) Feng, Y., Zheng, H., Zhu, Z. and Zu, F., The Microstructure and Electrical Conductivity of Aluminum Alloy Foams, Materials Chemistry and Physics, Vol. 78 (2002), pp. 196-201. 